\title{
Universality and Quantum Criticality of the One-Dimensional Spinor Bose Gas
}

\author{
Ovidiu I. Pâțu, ${ }^{1}$ Andreas Klümper, ${ }^{2}$ and Angela Foerster ${ }^{3}$ \\ ${ }^{1}$ Institute for Space Sciences, Bucharest-Măgurele, R 077125 Romania \\ ${ }^{2}$ Fakultät für Mathematik und Naturwissenschaften, Bergische Universität Wuppertal, 42097 Wuppertal, Germany \\ ${ }^{3}$ Instituto de Física da UFRGS, Avenida Bento Gonçalves 9500, Porto Alegre, Rio Grande do Sul, Brazil
}

(Received 21 November 2017; published 12 June 2018)

\begin{abstract}
We investigate the universal thermodynamics of the two-component one-dimensional Bose gas with contact interactions in the vicinity of the quantum critical point separating the vacuum and the ferromagnetic liquid regime. We find that the quantum critical region belongs to the universality class of the spin-degenerate impenetrable particle gas which, surprisingly, is very different from the singlecomponent case and identify its boundaries with the peaks of the specific heat. In addition, we show that the compressibility Wilson ratio, which quantifies the relative strength of thermal and quantum fluctuations, serves as a good discriminator of the quantum regimes near the quantum critical point. Remarkably, in the Tonks-Girardeau regime, the universal contact develops a pronounced minimum, reflected in a counterintuitive narrowing of the momentum distribution as we increase the temperature. This momentum reconstruction, also present at low and intermediate momenta, signals the transition from the ferromagnetic to the spin-incoherent Luttinger liquid phase and can be detected in current experiments with ultracold atomic gases in optical lattices.
\end{abstract}

DOI: $10.1103 /$ PhysRevLett.120.243402

Experiments with ultracold atomic gases allow for the creation and manipulation of various physical systems with an unprecedented degree of control over interaction strength, statistics of the constituent particles, and dimensionality [1]. This control materialized in the experimental realization of many low-dimensional systems whose physics is well captured by many-body integrable models allowing for a parameter free comparison between theory and experiment $[2,3]$. The need for accurate theoretical predictions is particularly stringent in low dimensions due to the enhancement of quantum fluctuations which invalidates our physical intuition based on the free-particle picture and mean-field theory. In the case of integrable models, powerful techniques associated with Bethe ansatz $[4,5]$ can be employed to analytically investigate the physical properties of such systems for all values of the interaction strength including the strong coupling limit.

One-dimensional (1D) multicomponent systems in which the constituent particles have a variable number of internal "pseudospin" states present very rich physics exhibiting quantum regimes not found in higher dimensions or in the case of their single-component counterparts $[2,3,6,7]$. As is well known, the single-component Bose gas in the Tonks-Girardeau regime with infinitely strong coupling realizes an impenetrable particle gas which is fully described by spinless free fermions. We see that the spinor gases in the very strong coupling regime behave very differently from free fermions.

At zero temperature, these systems present quantum phase transitions (QPT) which are driven by varying certain nonthermal parameters (chemical potential, magnetic field, pressure, etc.). In the vicinity of the quantum critical point (QCP), quantum and thermal fluctuations couple strongly characterizing the quantum critical (QC) regime [8]. In the phase space, the QC region has a distinct $V$-shape fanning out from the QCP $[8,9]$ at low temperatures, and in this region, the thermodynamics of the system is universal and scale invariant.

Motivated by the recent experimental confirmation [10] of quantum criticality in the Lieb-Liniger model [11], in this Letter, we theoretically investigate the more complex problem posed by the two-component generalization known as the spinor Bose gas [12-14]. We provide an analytical description of the universal thermodynamics and determine the location of the QCP, the critical exponents, and the boundaries of the critical region. The analytical description is derived for an arbitrary number of spin components. It is universally valid for bosons and fermions. It also explains the first order transition in the finite density regime with jump of the magnetization at a zero magnetic field. In addition, for the two-component Bose case, we perform a detailed analysis of the universal contact [15-18] and show that in the Tonks-Girardeau regime it presents a pronounced minimum, signaling the transition from the ferromagnetic [19-23] to the spin-incoherent liquid phase [24-26]. This transition is accompanied by a significant momentum reconstruction which can be experimentally detected.

We consider a system of 1D bosons with two internal "pseudospin" states denoted by $\uparrow$ and $\downarrow$ with spin-independent 
contact interactions. In the second quantization, the Hamiltonian is $H=\int \mathcal{H}(x) d x$ with density

$$
\begin{aligned}
\mathcal{H}(x)= & \sum_{\sigma=\{\uparrow, \downarrow\}}\left(\frac{\hbar^{2}}{2 m} \partial_{x} \Psi_{\sigma}^{\dagger}(x) \partial_{x} \Psi_{\sigma}(x)-\mu_{\sigma} \Psi_{\sigma}^{\dagger}(x) \Psi_{\sigma}(x)\right) \\
& +\frac{\hbar^{2} c}{2 m} \sum_{\sigma, \sigma^{\prime}=\{\uparrow, \downarrow\}} \Psi_{\sigma}^{\dagger}(x) \Psi_{\sigma^{\prime}}^{\dagger}(x) \Psi_{\sigma^{\prime}}(x) \Psi_{\sigma}(x)
\end{aligned}
$$

where $\Psi_{\sigma}(x), \Psi_{\sigma}^{\dagger}(x)$ are bosonic fields satisfying $\left[\Psi_{\sigma}(x)\right.$, $\left.\Psi_{\sigma^{\prime}}^{\dagger}(y)\right]=\delta_{\sigma, \sigma^{\prime}} \delta(x-y), m$ is the mass of the particles, $\mu_{\sigma}$ are chemical potentials, and the coupling constant can be expressed in terms of the 1D scattering length via $c=-2 / a_{1 D}$ [27]. In the following, it is useful to introduce $\mu=\left(\mu_{\uparrow}+\mu_{\downarrow}\right) / 2$ and $H=\left(\mu_{\uparrow}-\mu_{\downarrow}\right) / 2$. In units of $\hbar=$ $2 m=1$, the dimensionless coupling constant is $\gamma=c / n$ with $n$ the total density, and the Fermi temperature is $T_{F}=\pi^{2} n^{2}$. The system is weakly (strongly) interacting when $\gamma \ll 1(\gamma \gg 1)$.

The Hamiltonian (1) is integrable $[12,13]$ and the ground state and low-lying excitations were derived using the nested Bethe ansatz [14]. Compared with the fermionic model, for which the ground state is antiferromagnetic, in this case, the ground state is fully polarized (ferromagnetic) which is a general characteristic of bosonic 1D models with spin-independent interactions [28]. At low temperatures, the longitudinal low-lying excitations are plasmons $(\epsilon(p) \sim|p|)$, but the softest transversal low-lying excitation is a magnon with quadratic dispersion $\lim _{p \rightarrow 0} \epsilon(p) \sim$ $p^{2} / 2 m_{*}$ which prevents the use of Luttinger liquid (LL) theory $\left(m_{*}=3 m \gamma / 2 \pi^{2}\right.$ for large $\gamma$ [29]).

Computing the thermodynamic properties of the spinor Bose gas is a notoriously difficult task despite the integrability of the Hamiltonian (1). In contrast with the single component case (the Lieb-Liniger model), the thermodynamic Bethe ansatz (TBA) [30,31] description of the multicomponent model [32] involves an infinite number of nonlinear integral equations (NLIEs). Even though numerical schemes can be developed to approximately solve such systems [33-35], they are rather cumbersome and require truncations in the number of equations which can introduce uncontrollable errors. In [36,37], two of the authors derived an efficient thermodynamic description which made use of the lattice embedding of the spinor Bose gas in the $q=3$ Perk-Schultz spin chain and the quantum transfer matrix $[5,38,39]$. In this description, the grandcanonical potential per length is given by $\phi(\mu, H, T)=$ $-(T / 2 \pi) \int_{-\infty}^{+\infty} \ln \left[1+a_{1}(k)\right]+\ln \left[1+a_{2}(k)\right] d k$, with $a_{i}(k)$ auxiliary functions satisfying the following system of NLIEs:

$$
\begin{aligned}
\ln a_{1}(k)= & -\left(k^{2}-\mu-H\right) / T \\
& +\left(K_{0} * \ln A_{1}\right)(k)+\left(K_{2} * \ln A_{2}\right)(k-i \epsilon),
\end{aligned}
$$

$$
\begin{aligned}
\ln a_{2}(k)= & -\left(k^{2}-\mu+H\right) / T \\
& +\left(K_{1} * \ln A_{1}\right)(k+i \epsilon)+\left(K_{0} * \ln A_{2}\right)(k),
\end{aligned}
$$

where $A_{i}(k)=1+a_{i}(k), K_{0}(k)=2 c /\left(k^{2}+c^{2}\right), K_{1}(k)=$ $c /[k(k+i c)], \quad K_{2}(k)=c /[k(k-i c)], \quad$ and $[f * g](k)=$ $(1 / 2 \pi) \int_{-\infty}^{+\infty} f\left(k-k^{\prime}\right) g\left(k^{\prime}\right) d k^{\prime}$. We stress that Eqs. (2) are valid for all values of $\mu, H, T$, and $c$, and they can be easily and efficiently numerically implemented allowing for the exploration of certain regions of the relevant parameters space (like $H \ll T, \mu, c$ ) which are inaccessible by TBA. These equations constitute the basis of our investigations.

At $T=0$ and fixed $H$, the spinor Bose gas presents a QPT between the vacuum and ferromagnetic liquid phase when the chemical potential reaches the QCP. In the vicinity of the QCP, the thermodynamics of the system is universal and scale invariant and can be described by $[40,41]$

$p(\mu, H, T) \sim p_{r}(\mu, H, T)+T^{(d / z)+1} \mathcal{P}_{H}\left(\frac{\mu-\mu_{c}(H)}{T^{(1 / \nu z)}}\right)$

where $p=-\phi$ is the pressure, $p_{r}$ the regular part, $z$ the dynamical critical exponent, $\nu$ the correlation length exponent, $d=1$ the dimension, and $\mathcal{P}_{H}$ is a universal function describing the singular part of the pressure. The determination of the universality class (the values of $z$ and $\nu$ ) and of the universal function is accomplished by performing the following steps [40]. (i) Choose $z$ and $\nu$. (ii) For these values of $z$ and $\nu$, plot for several values of temperature the "scaled pressure" $\left(p-p_{r}\right) T^{-(d / z)-1}$ (at low $T p_{r}$ can be replaced by a constant which in our case is zero). If the critical exponents are chosen correctly, then all plots should intersect at $\mu_{c}(H)$ identifying the QCP. (iii) With $z, \nu$, and $\mu_{c}(H)$ determined, the plots of the "scaled pressures" at all temperatures as functions of $\left(\mu-\mu_{c}(H)\right) / T^{(1 / \nu z)}$ will collapse to a single curve which is the universal function $\mathcal{P}_{H}$.

Our investigations reveal [42] lines of QCPs at $\mu_{c}(H)=$ $-|H|$ with critical exponents $z=2$ and $\nu=1 / 2$. We identify the universality class as the spin-degenerate impenetrable particle gas. This can be understood intuitively by an elementary, yet quantitative derivation. For any repulsive interaction, at low densities, the system is in the strong coupling regime with finite energy states realized by wave functions $\psi\left(x_{1}, \ldots, x_{N}, s_{1}, \ldots, s_{N}\right)$ that vanish if the spatial coordinates of any two particles coincide. We construct such wave functions by first considering the fundamental regime with order of spatial coordinates $x_{1}<x_{2}<\cdots<x_{N}$. Here, we use the product ansatz $\phi$. $\chi$ where $\phi\left(x_{1}, \ldots, x_{N}\right)$ is a Slater determinant of plane waves with pairwise different wave numbers $k_{1}, \ldots, k_{N}$, and $\chi=\left|\sigma_{1}, \ldots, \sigma_{N}\right\rangle$ is an arbitrary spin part. For all other sectors with different order of spatial coordinates, we reorder simultaneously all spatial and spin coordinates 
leading to the fundamental regime and then invoke the symmetry of the total wave function.

The energy of the product states is given by the sum of the kinetic energy terms $\left(k^{2}\right)$ and the Zeeman terms $( \pm H)$. We set up the partition function of the grand-canonical ensemble. The space of configurations consists of all combinations of occupations of $k$ modes: empty and singly occupied with spin up or spin down. The partition function is a product over characteristic factors $Z=$ $\prod_{k}\left[1+e^{-\beta\left(k^{2}-\mu-H\right)}+e^{-\beta\left(k^{2}-\mu+H\right)}\right]$ for all $k$ values allowed by the boundary conditions. Note that this derivation with exactly the same result also works for fermions with infinitely strong repulsion. The generalization to arbitrary spin numbers is obvious.

The universal thermodynamics (for both fermions and bosons) in the vicinity of the QCP is given by Takahashi's formula [31] $[x=(\mu+|H|) / T, y=H / T]$,

$$
p=\frac{T^{3 / 2}}{2 \pi} \int_{-\infty}^{+\infty} \ln \left[1+\left(1+e^{-2|y|}\right) e^{-k^{2}+x}\right] d k
$$

Figure 1 shows the collapse of Wilson ratio (see below) data onto the universal function analytically derived from Eq. (4). Also, the structures of the QC regime are fully described by Eq. (4), see Fig. 2. Despite its apparent simplicity, the spindegenerate impenetrable particle gas has a rich phase diagram and surprising properties. The critical exponents suggest that the system might be equivalent to free
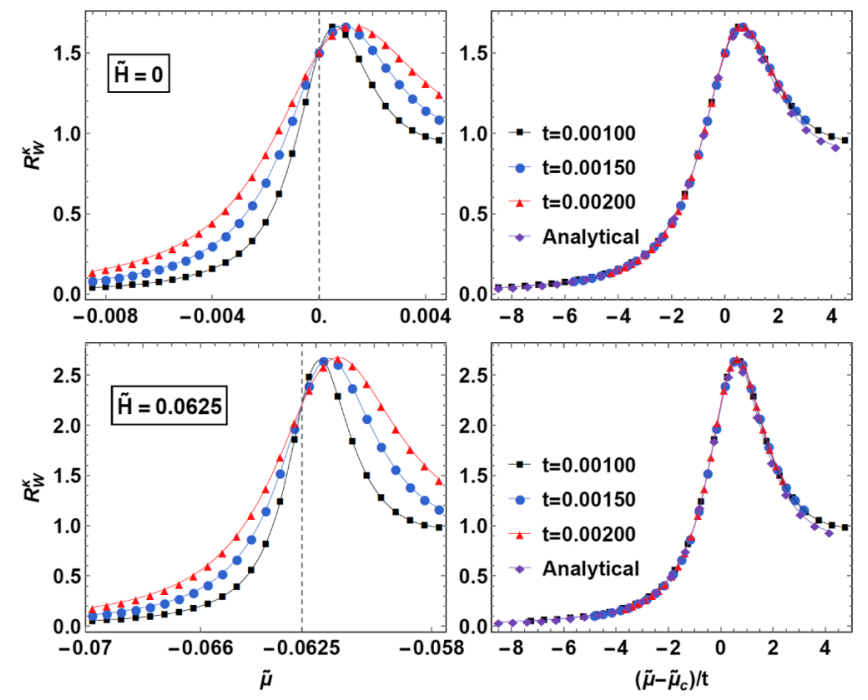

FIG. 1. Scaling behavior of the compressibility Wilson ratio $R_{W}^{\kappa}$ for $\tilde{H}=0$ (upper panels) and $\tilde{H}=0.0625$ (lower panels) $\left(\tilde{H}=H / c^{2}, \tilde{\mu}=\mu / c^{2}, t=T / c^{2}\right.$ with $\left.c=2\right)$. The curves at different temperatures intersect at QCP $\tilde{\mu}_{c}=-|\tilde{H}|$. Plots of the ratio as a function of $\left(\tilde{\mu}-\tilde{\mu}_{c}\right) / t$ reveal the universal function $\mathcal{Q}_{H}(x)$. In the right panels, the magenta diamonds represent the analytical predictions given by Eq. (4) with $e^{-|y|}=0$ for $H \neq 0$ and $e^{-|y|}=1$ for $H=0$.
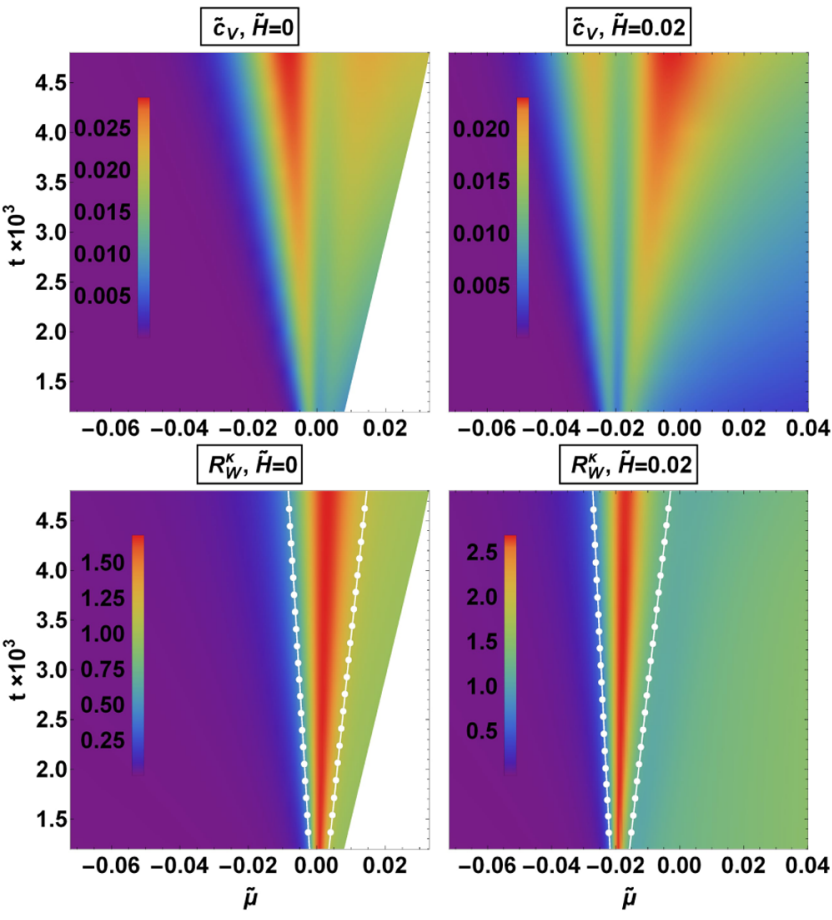

FIG. 2. Density plots of the grand-canonical specific heat $\tilde{c}_{V}=$ $c_{V} / c$ (upper panels) and Wilson ratio (lower panels) for $\tilde{H}=0$ and $\tilde{H}=0.02(c=5)$. The specific heat presents maxima fanning out from the critical value of the chemical potential $\mu_{c}=$ $-|H|$ which determine the boundaries of the QC region. The white lines in the Wilson ratio plots are the boundaries of the QC region given by the maxima of the specific heat. To the left of the boundary lies the vacuum phase at finite temperature which can be well approximated by a classical gas and to the right the ferromagnetic liquid regime.

fermions with the pressure given by $\left(x^{\prime}=\mu / T\right), p_{\mathrm{FF}}=$ $\left(T^{3 / 2} / 2 \pi\right) \int \ln \left[\left(1+e^{-k^{2}+x^{\prime}+y}\right)\left(1+e^{-k^{2}+x^{\prime}-y}\right)\right] d k$. However, free fermions have a $\mu-H$ phase diagram with four phases (vacuum, spin-up particles, spin-down particles, and a mixture of spin-up and spin-down particles) and critical lines $\mu= \pm H$. In contrast, the spin-degenerate impenetrable particle gas has only three phases (vacuum, spin-up particles, and spin-down particles) with critical lines $\mu=-|H|$ and $\mu>0, H=0$. At the latter line, first order transitions take place. Here, simply by taking the $H$ derivative of Eq. (4) (see also [42]), we find a magnetization with jump at zero field $m \sim \operatorname{sgn} H(\mu+|H|)^{1 / 2} / \pi$, and at $T=H=0$ also residual entropy $s \sim \mu^{1 / 2} \ln 2 / \pi$ ! Despite the deceiving familiar values of the critical exponents, the physics of the spin-degenerate impenetrable particle gas is drastically different from that of ordinary free fermions.

It was shown in [43-45] that the susceptibility and compressibility Wilson ratios can serve as good discriminators of quantum phases in attractive multicomponent ultracold gases. In our case, the relevant quantity is the compressibility Wilson ratio defined by $R_{W}^{\kappa}=\left(\pi^{2} k_{B}^{2} / 3\right) T\left(\kappa / c_{V}\right)$ 
with $\kappa=-\partial^{2} \phi / \partial \mu^{2}$ the compressibility and $c_{V}=$ $-T \partial^{2} \phi / \partial T^{2}$ the grand-canonical specific heat at constant volume. In the vicinity of the QCP, the Wilson ratio scales like $R_{W}^{\kappa} \sim \mathcal{Q}_{H}\left[\left(\mu-\mu_{c}(H)\right) / T\right]$ with $\mathcal{Q}_{H}(x)=\mathcal{P}_{H}^{\prime \prime}(x) /$ $\left(\frac{3}{4} \mathcal{P}_{H}(x)-x \mathcal{P}_{H}^{\prime}(x)+x^{2} \mathcal{P}_{H}^{\prime \prime}(x)\right)$ a universal function. In the vacuum phase, $R_{W}^{\kappa}$ is zero and presents a sudden enhancement in the $\mathrm{QC}$ region becoming almost constant in the ferromagnetic liquid phase. Taking into account that in the grand-canonical ensemble the compressibility and specific heat quantify the energy and particle fluctuations via $k_{B} T \kappa=\left\langle\delta N^{2}\right\rangle$ and $k_{B} T^{2} c_{V}=\left\langle\delta(E-\mu N)^{2}\right\rangle$, it is easy to understand why $R_{W}^{\kappa}$ identifies the quantum regimes so efficiently. In the critical regime, the thermal and quantum fluctuations couple strongly resulting in the anomalous enhancement in the vicinity of the QCP. In the upper panels of Fig. 2, we show that the specific heat presents a continuous set of local maxima on both sides of the QCP, peaks that can be identified with the boundaries of the QC region (see also $[10,45,46]$ ). Depicting these boundaries in the density plots of the Wilson ratio (lower panels of Fig. 2), we see that they clearly delimitate the three quantum regimes.

A universal property of physical systems with contact interactions is that the large momentum distribution behaves like $\lim _{k \rightarrow \infty} n_{\sigma} \sim \mathcal{C}_{\sigma} / k^{4}$ with $\mathcal{C}_{\sigma}$ the amplitude called contact [15-18,47]. In the case of the bosonic Gaudin-Yang model, the total contact can be computed from the thermodynamics [47] as $\mathcal{C}=\mathcal{C}_{\uparrow}+\mathcal{C}_{\downarrow}=$ $c^{2} \partial^{2} \phi / \partial c^{2}$. In Fig. 3, we show the temperature dependence of the dimensionless total contact $s=\mathcal{C} / k_{F}^{4}$ with $k_{F}=\pi n$
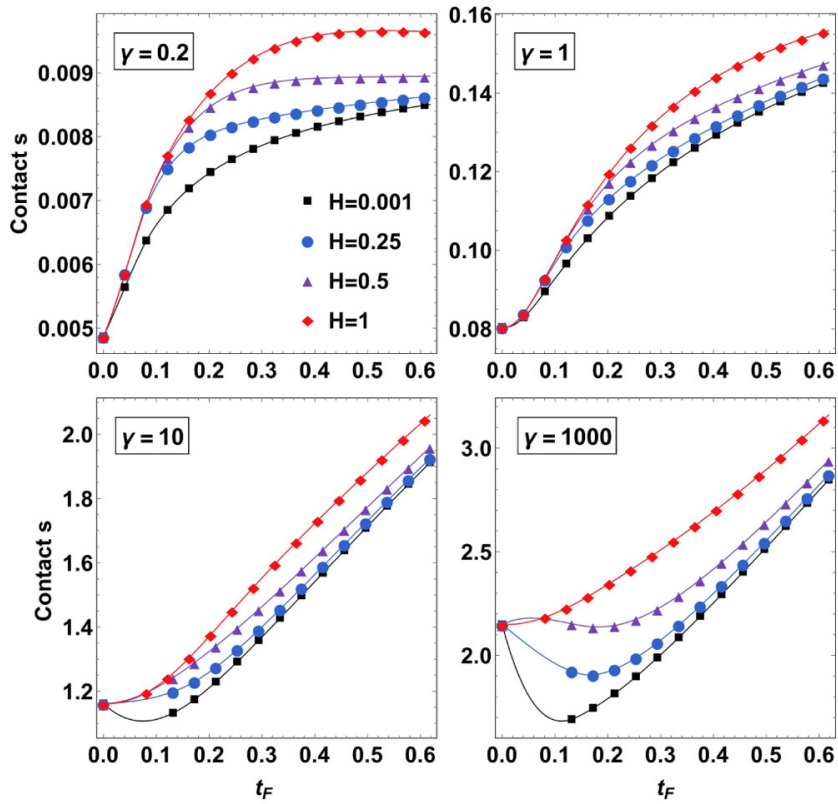

FIG. 3. Dependence of the dimensionless total contact $s$ on the temperature $\left(t_{F}=T / T_{F}, \quad \gamma=c / n, \quad n=1 / 2\right)$ for $\gamma=$ $\{0.2,1,10,1000\}$ and several values of $H$. At strong coupling and low $H$, the contact develops a minimum. for several values of coupling constant and magnetic field. For $\gamma=\{0.2,1\}$, it is a monotonically increasing function of the temperature for all values of $H$. At strong coupling, the contact develops a minimum at low temperatures which is more pronounced as $H$ decreases. This means that for $\gamma \gg 1$, we encounter the counterintuitive phenomenon in which the fraction of particles with high momenta decreases as we increase the temperature. It was first noticed in [48] for the case of impenetrable particles at $H=0$ that this momentum reconstruction signals the
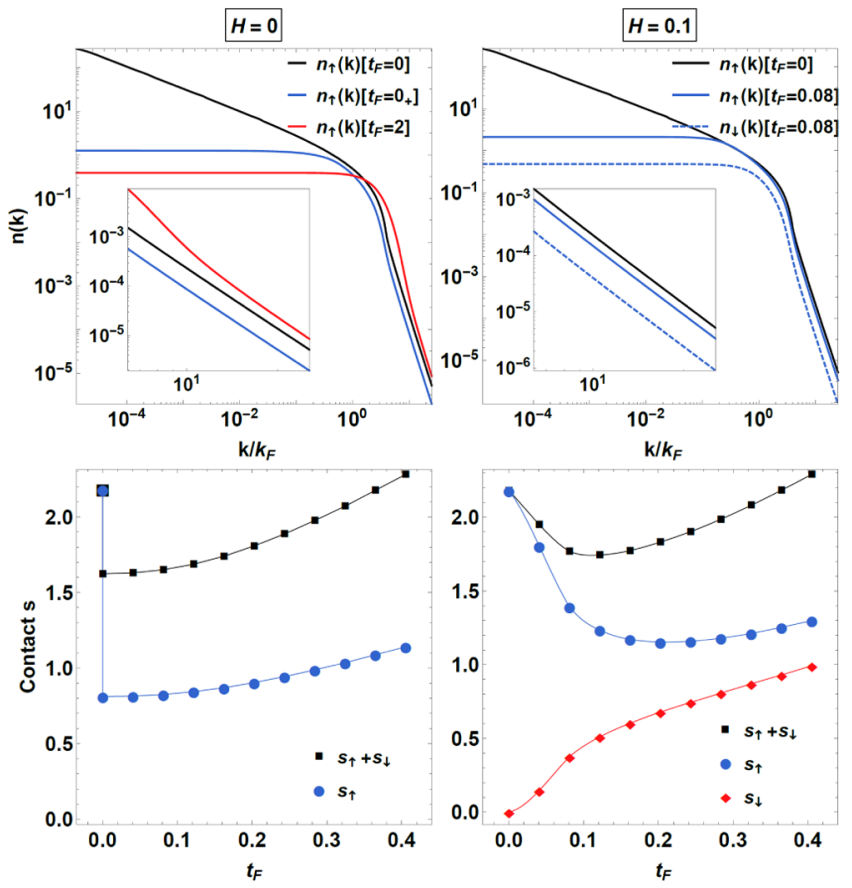

FIG. 4. Upper left panel: Momentum distribution in log-log coordinates for the balanced impenetrable gas $(H=0)$ for $t_{F}=\left\{0,0_{+}, 2\right\}$. For the balanced system at a fixed temperature, $n_{\uparrow}(k)=n_{\downarrow}(k)$. Data show a significant momentum reconstruction especially at low and high momenta as we increase the temperature from the ferromagnetic liquid $\left(t_{F}=0\right)$ to the spinincoherent LL regime $\left(t_{F}=0_{+}\right)$. The inset shows the high momentum tail from which the contact can be extracted and shows that $s_{\uparrow}\left(t_{F}=0\right)>s_{\uparrow}\left(t_{F}=0_{+}\right)$. Lower left panel: Dependence of the dimensionless total contact $s_{\uparrow}+s_{\downarrow}$ (black squares) and contact of spin-up particles $s_{\uparrow}$ (blue disks) as functions of the temperature for the balanced system. While at $t_{F}=0$ the ground state is populated only by type 1 particles, at $t_{F}=0_{+}$the system is balanced and $s_{\uparrow}=s_{\downarrow}$. The total contact and also $s_{\uparrow}$ present a sharp minimum at $t_{F}=0_{+}$. Upper right panel: Momentum distribution for $H=0.1$ and $t_{F}=\{0,0.08\}$. In this case, for a fixed temperature, $n_{\uparrow}(k) \neq n_{\downarrow}(k)$. Data also show a significant momentum reconstruction but not as pronounced as in the balanced case. The inset shows that $s_{\uparrow}\left(t_{F}=0\right)>s_{\uparrow}\left(t_{F}=0.08\right)>$ $s_{\downarrow}\left(t_{F}=0.08\right)$. Lower right panel: Temperature dependence of the contact for $H=0.1$. Even though $s_{\downarrow}$ is monotonically increasing, the total contact and $s_{\uparrow}$ present a local minimum at low temperatures. In all cases, $n=1 / 2$. 
transition from the ferromagnetic liquid phase $\left(T<T_{0} \sim\right.$ $\left.T_{F} / \gamma\right)$ to the spin-incoherent LL regime $\left(T_{0}<T<T_{F}\right)$ [24-26]. The two temperature scales become well separated for $\gamma \gg 1$ which explains why we encounter this phenomenon only in the strong coupling limit.

In the impenetrable case, the momentum distribution and the contact of each species of particles can be computed using the Fredholm determinant representation for the Green's function $\left\langle\Psi_{i}^{\dagger}(x) \Psi_{i}(0)\right\rangle_{T, \mu, H}$ obtained in [49]. Its Fourier transform yields the momentum distribution $n_{i}(k)$, and the contact is extracted as $C_{i}=\lim _{k \rightarrow \infty} n_{i}(k) / k^{4}$. The detailed results in Fig. 4 reveal in addition to the high momentum crossover also a significant momentum reconstruction at low momenta. This reconstruction, which takes place in a very narrow interval of temperature, is largest for the balanced system (see the left panels of Fig. 4), and becomes more attenuated as we increase $H$. The low-momentum crossover has a simple explanation. At $T=H=0$, the system is described by the impenetrable Lieb-Liniger model for which the ground state is a quasicondensate with $n_{\uparrow}(k) \sim 1 / \sqrt{k}$ for small $k$ (corresponding to the large distance asymptotics of the Green's function $\sim 1 / x^{1 / 2}$ ). As we increase the temperature, the system is described by the spin-incoherent LL with exponentially decreasing Green's function at large distances [48] and a finite momentum distribution at low momenta. The same type of reasoning also holds for strong but finite coupling implying that the momentum reconstruction both at low and high momenta can be experimentally observed even for moderate values of $\gamma$.

In summary, we have investigated the universal properties of the spinor Bose gas and identified the universality class and boundaries of the QC region separating the vacuum from the ferromagnetic liquid phase. The universal contact increases with increasing magnetic field opposite to the behavior of fermionic systems. In the Tonks-Girardeau regime, the contact develops a pronounced minimum indicating a significant momentum reconstruction at low temperatures which can be detected in current experiments with ultracold gases.

O.I. P. acknowledges the financial support from the LAPLAS 4 and LAPLAS 5 programs of the Romanian National Authority for Scientific Research. A. K. is grateful to DFG (Deutsche Forschungsgemeinschaft) for financial support in the framework of the research unit FOR 2316. A. F. acknowledges CNPq (Conselho Nacional de Desenvolvimento Científico e Tecnológico) for financial support. All authors would like to thank the mathematical research institute MATRIX in Australia where part of this research was performed.

[1] I. Bloch, J. Dalibard, and W. Zwerger, Rev. Mod. Phys. 80, 885 (2008).
[2] M. A. Cazalilla, R. Citro, T. Giamarchi, E. Orignac, and M. Rigol, Rev. Mod. Phys. 83, 1405 (2011).

[3] X.-W. Guan, M. T. Batchelor, and C. Lee, Rev. Mod. Phys. 85, 1633 (2013).

[4] V. E. Korepin, N. M. Bogoliubov, and A. G. Izergin, Quantum Inverse Scattering Method and Correlation Functions (Cambridge University Press, Cambridge, England, 1993).

[5] F. H. L. Essler, H. Frahm, F. Göhmann, A. Klümper, and V.E. Korepin, The One-Dimensional Hubbard Model (Cambridge University Press, Cambridge, England, 2005).

[6] P. Wicke, S. Whitlock, and N. J. van Druten, arXiv:1010 .4545 .

[7] G. Pagano, M. Mancini, G. Cappellini, P. Lombardi, F. Schäfer, H. Hu, X.-J. Liu, J. Catani, C. Sias, M. Inguscio, and L. Fallani, Nat. Phys. 10, 198 (2014).

[8] S. Sachdev, Quantum Phase Transitions (Cambridge University Press, Cambridge, England, 2011).

[9] P. Coleman and A. J. Schofield, Nature (London) 433, 226 (2005).

[10] B. Yang, Y.-Y. Chen, Y.-G. Zheng, H. Sun, H.-N. Dai, X.-W. Guan, Z.-S. Yuan, and J.-W. Pan, Phys. Rev. Lett. 119, 165701 (2017).

[11] E. H. Lieb and W. Liniger, Phys. Rev. 130, 1605 (1963).

[12] C. N. Yang, Phys. Rev. Lett. 19, 1312 (1967).

[13] M. Gaudin, Phys. Lett. A 24, 55 (1967).

[14] Y.-Q. Li, S.-J. Gu, Z.-J. Ying, and U. Eckern, Europhys. Lett. 61, 368 (2003).

[15] S. Tan, Ann. Phys. (Amsterdam) 323, 2952 (2008); 323, 2971 (2008); 323, 2987 (2008).

[16] M. Olshanii and V. Dunjko, Phys. Rev. Lett. 91, 090401 (2003).

[17] M. Barth and W. Zwerger, Ann. Phys. (Amsterdam) 326, 2544 (2011).

[18] Y.-Y. Chen, Y.-Z. Jiang, X.-W. Guan, and Q. Zhou, Nat. Commun. 5, 5140 (2014).

[19] M. B. Zvonarev, V. V. Cheianov, and T. Giamarchi, Phys. Rev. Lett. 99, 240404 (2007).

[20] S. Akhanjee and Y. Tserkovnyak, Phys. Rev. B 76, 140408 (R) (2007).

[21] K. A. Matveev and A. Furusaki, Phys. Rev. Lett. 101, 170403 (2008).

[22] A. Kamenev and L. I. Glazman, Phys. Rev. A 80, 011603(R) (2009).

[23] M. B. Zvonarev, V. V. Cheianov, and T. Giamarchi, Phys. Rev. B 80, 201102(R) (2009).

[24] V. V. Cheianov and M. B. Zvonarev, Phys. Rev. Lett. 92, 176401 (2004).

[25] G. A. Fiete and L. Balents, Phys. Rev. Lett. 93, 226401 (2004).

[26] G. A. Fiete, Rev. Mod. Phys. 79, 801 (2007).

[27] M. Olshanii, Phys. Rev. Lett. 81, 938 (1998).

[28] E. Eisenberg and E. H. Lieb, Phys. Rev. Lett. 89, 220403 (2002).

[29] J. N. Fuchs, D. M. Gangardt, T. Keilmann, and G. V. Shlyapnikov, Phys. Rev. Lett. 95, 150402 (2005).

[30] C. N. Yang and C. P. Yang, J. Math. Phys. (N.Y.) 10, 1115 (1969)

[31] M. Takahashi, Thermodynamics of One-Dimensional Solvable Models (Cambridge University Press, Cambridge, England, 1999). 
[32] S. J. Gu, Y. Q. Li, Z. J. Ying, and X. A. Zhao, Int. J. Mod. Phys. A 16, 2137 (2002).

[33] J. S. Caux, A. Klauser, and J. van den Brink, Phys. Rev. A 80, 061605(R) (2009).

[34] A. Klauser and J.-S. Caux, Phys. Rev. A 84, 033604 (2011).

[35] X. W. Guan, M. T. Batchelor, and M. Takahashi, Phys. Rev. A 76, 043617 (2007).

[36] A. Klümper and O. I. Pâţu, Phys. Rev. A 84, 051604(R) (2011).

[37] O. I. Pâţu and A. Klümper, Phys. Rev. A 92, 043631 (2015).

[38] A. Klümper, Ann. Phys. (Berlin) 504, 540 (1992).

[39] A. Klümper, Z. Phys. B 91, 507 (1993).

[40] Q. Zhou and T.-L. Ho, Phys. Rev. Lett. 105, 245702 (2010).

[41] M. P. A. Fisher, P. B. Weichman, G. Grinstein, and D. S. Fisher, Phys. Rev. B 40, 546 (1989).

[42] See the Supplemental Material at http://link.aps.org/ supplemental/10.1103/PhysRevLett.120.243402 for the scaling behavior of the pressure and density, asymptotic analysis of the universal thermodynamics, and derivation of the magnetization and entropy.

[43] X.-W. Guan, X.-G. Yin, A. Foerster, M. T. Batchelor, C.-H. Lee, and H.-Q. Lin, Phys. Rev. Lett. 111, 130401 (2013).

[44] Y.-C. Yu, Y.-Y.Chen, H.-Q. Lin, R. A. Römer, and X.-W. Guan, Phys. Rev. B 94, 195129 (2016).

[45] F. He, Y.-Z. Jiang, Y.-C. Yu, H.-Q. Lin, and X.-W. Guan, Phys. Rev. B 96, 220401 (2017).

[46] Y. Maeda, C. Hotta, and M. Oshikawa, Phys. Rev. Lett. 99, 057205 (2007).

[47] O. I. Pâţu and A. Klümper, Phys. Rev. A 96, 063612 (2017).

[48] V. V. Cheianov, H. Smith, and M. B. Zvonarev, Phys. Rev. A 71, 033610 (2005).

[49] A. G. Izergin and A. G. Pronko, Nucl. Phys. B520, 594 (1998). 